\title{
Adecuación Curricular para Incremento del Vocabulario del Idioma Inglés en los Estudiantes de Octavo Año de Guayaquil
}

\section{Curricular Adaptation to Increase the Vocabulary in English for Eighth-grade Students from Guayaquil}

\author{
Israel Bravo Bravo \\ Universidad de Guayaquil \\ Guayaquil, Ecuador \\ israel.bravob@ug.edu.ec \\ Orcid: 0000-0003-0648-4061
}

\author{
Maria Palmira Alves \\ Universidad de Minho \\ Braga, Portugal \\ palves@ie.uminho.pt \\ Orcid: 0000-0002-3108-744X
}

\begin{abstract}
Resumen - Esta investigación tiene como propósito principal analizar la incidencia de la adecuación curricular en el incremento del vocabulario del idioma inglés en estudiantes de octavo año Educación General Básica (EGB) de las instituciones educativas fiscales de Guayaquil. Para ello, es necesario describir varios factores tales como la falta de un currículo del Inglés en la primaria que facilite una base sólida de conocimientos de este idioma, el contenido académico muy superior en comparación a los años de primaria. Se enfoca, principalmente, en el diseño curricular que rige en la actualidad para estudiar cómo su aplicación determina el rendimiento académico en cuanto a la adquisición de vocabulario y, con ello, el aprendizaje y dominio del idioma por parte de los estudiantes. El estudio se desarrolla bajo una metodología investigativa de campo, transversal, no experimental y descriptiva, con un enfoque cuantitativo, y con un diseño tipo encuesta, para evaluar la información obtenida a través de un cuestionario de 24 preguntas aplicado a 469 profesores del área de inglés del octavo año pertenecientes a las instituciones educativas fiscales de Guayaquil. Los resultados muestran una baja eficiencia en la adquisición de vocabulario por parte de los estudiantes debido a la falta de adecuación curricular. Se concluye que para mejorar el aprendizaje del inglés es necesario adaptar el currículo a las necesidades individuales, recomendándose la aplicación de un diagnóstico previo del campo lexical en los estudiantes.
\end{abstract}

Palabras Clave: Currículo, Vocabulario, Aprendizaje Inglés.

\begin{abstract}
The main purpose of this research is to analyze the incidence of curricular adaptation towards the increase of the English language vocabulary in eighth grade BGE students of public education in Guayaquil city. It was necessary to describe some factors such as the lack of an English curriculum in primary school to provide a solid base of knowledge of that language, the higher academic content compared to the primary school years. The focus was mainly on the curricular design currently applied to study how its application determines the academic performance in terms of vocabulary acquisition and the students' language learning and mastery. The study was developed under a crosssectional, non-experimental, and descriptive field research methodology, with a quantitative approach. A survey-type tool was designed to evaluate the information, and the 24-question questionnaire was applied to 469 eighth-grade English teachers from public education in Guayaquil city. The findings showed low efficiency in vocabulary acquisition in those students due to the lack of a curricular adaptation. This research concludes that English learning improvement in Guayaquil city should be based on a curriculum adaptation. The application of a previous diagnosis of the Lexi level is our recommendation.
\end{abstract}

Keywords: Curriculum, Vocabulary, English Learning

Sumario: I Introducción, II Desarrollo, III Metodología, IV Resultados, V Discusión, VI Conclusiones.

Como citar: Bravo, Israel., Alves, Maria. (2020). Adecuación Curricular para Incremento del Vocabulario del Idioma Inglés en los Estudiantes de Octavo Año de Guayaquil. Revista Tecnológica - Espol, 32(2). Recuperado a partir de http://www.rte.espol.edu.ec/index.php/tecnologica/article/view/781 


\section{INTRODUCCIÓN}

De acuerdo con [1] el idioma inglés es considerado como la lengua más hablada en el mundo, esto ha ocasionado que las instituciones educativas del país lo integren en el currículum educativo. Al referirse al inglés como un idioma extranjero diferente al nativo es necesario considerar el proceso de aprendizaje que esta lengua requiere y que normalmente el estudiante no aplica en su vida diaria. Motivo por el cual, el proceso de aprendizaje del inglés se realiza dentro de las aulas de clase, donde se utilizan diversas actividades y ejercicios para monitorear el desempeño del estudiante en este idioma.

Tal como lo menciona [2], con la actual globalización el idioma inglés se ha convertido en un factor muy importante para el desarrollo de los países, dando lugar a que se ponga énfasis en un diseño curricular que potencie el aprendizaje en la masa estudiantil.

Sin embargo, según [3], existe la tendencia a incorporar en el currículo más elementos vinculados a la gramática que aquellos orientados a la ampliación del vocabulario, obviando el hecho de que este es fundamental para la adquisición, comprensión y producción del idioma. Tal tendencia ha conllevado a que en la planificación curricular en el aula generalmente prevalezca una metodología de enseñanza teórica por encima de la práctica.

Como resultado de lo anterior en las instituciones educativas del país la enseñanza del idioma inglés estaría mucho más enfocada en estructuras gramaticales; lo que estaría derivando hacia deficiencias en el aprendizaje del inglés.

Al respecto, [4] expresa que para aprender un idioma diferente al nativo se requiere perseverancia, atención, compromiso y mucho tiempo e interés, y que para diseñar un currículo educativo para un idioma extranjero, el docente se encuentra con grandes desafíos como la elección de una metodología y secuencia de enseñanza equitativa entre el vocabulario y estructuras gramaticales, donde existen varias recomendaciones, para lo que se debe analizar detenidamente los conocimientos previos de la audiencia.

En el caso del vocabulario, [4] menciona que es necesario investigar la frecuencia de las palabras en el habla, aunque también se debe de considerar lo preciso de un vocablo y la semejanza con otros, por lo cual es esencial incluir palabras que permitan definirlas.

En consecuencia, se deben definir y precisar los contenidos a impartir a los estudiantes en el proceso de enseñanza-aprendizaje del idioma inglés, tanto en vocabulario como en gramática, de acuerdo a su entorno y las necesidades que el mismo le exige, es decir en su uso práctico. Por ello, se debe adecuar el currículo a tal fin que se aproxime a la realidad del estudiante.

Por todos estos motivos, se aborda este estudio investigativo con el propósito de determinar la relación entre el nivel de inclusión en el diseño curricular de contenidos, estrategias y actividades dirigidas a la ampliación del vocabulario y el aprendizaje del idioma inglés de los estudiantes de octavo año Educación General Básica (EGB) de colegios fiscales.

\section{A. Planteamiento del tema}

El dominio de la lengua inglesa para [5] cada día es más solicitado en los negocios y las relaciones internacionales; bajo esta línea, se relaciona con factores de progreso y crecimiento económico en escala global. El interés por adquirir la habilidad de hablar el idioma inglés se extiende por toda Latinoamérica. Esta región ha realizado enormes esfuerzos para mejorar el proceso de enseñanzaaprendizaje de esta lengua a través de políticas y programas, lo que ha generado que cada vez más personas en la región puedan acceder al aprendizaje del inglés.

De acuerdo con [4] en Ecuador, el "Ministerio de Educación" a través de la "Dirección Nacional de Currículo y la División Nacional de Idiomas Extranjeros", ha definido nuevos lineamientos y ajustes en los currículos de inglés para el octavo, noveno y décimo año de Educación General Básica, de igual manera lo hizo con el primero, segundo y tercero de bachillerato, convencido de la necesidad de mejorar la enseñanza del inglés.

En esencia, se considera a este idioma como una herramienta indispensable para crear profesionales más competentes en el futuro por lo que, hoy en día, se deben preparar contenidos de calidad para enseñar inglés en las aulas a los estudiantes y, por tal motivo, se le debe incluir en el currículo y dar la debida importancia como parte de una educación integral.

En la provincia del Guayas el idioma inglés posee una importancia significativa, debido a que ha generado gran interés en la población como consecuencia de la globalización, lo que provoca que las ciudades entren a un proceso de desarrollo tecnológico dado que se hace fundamental el empleo de idiomas que vayan acorde con la nueva sociedad globalizante.

En el caso de la ciudad de Guayaquil existe una tendencia a la actualización de los contenidos académicos con el fin de promover el proceso de enseñanzaaprendizaje del idioma inglés, especialmente en las instituciones educativas fiscales. Sin embargo, tal como señala [4], generalmente los profesores que dan cátedra del idioma inglés ejecutan la planificación curricular de manera rígida, sin considerar las necesidades del alumno y su entorno.

Uno de los inconvenientes de este enfoque rígido en la planificación curricular es que a menudo se obvia la adquisición de vocabulario, concediéndole más espacios y contenidos a los tópicos gramaticales, por ejemplo. Esta situación se da a pesar de que la ampliación del vocabulario es esencial para el aprendizaje de una lengua, pues "Si las estructuras de la lengua constituyen el esqueleto de la lengua, entonces es el vocabulario el que proporciona los órganos vitales y la carne" [6].

Contar con un repertorio léxico abundante resulta importante, porque esto ayuda al estudiante a leer y hablar con mayor fluidez, usando el contexto para deducir el significado de las nuevas palabras que se le presenten [3]. Otra razón que al contar con un mayor campo de conocimiento sobre el vocabulario el/la estudiante aprende y reconoce con mayor facilidad las estructuras gramaticales, lo que le permitirá escribir y leer con confianza ya que no teme encontrarse con palabras nuevas [3].

En este sentido, si se espera que el estudiante aprenda inglés es necesario transcender el aprendizaje tradicionalista y profundizar en la adecuación curricular, pues adaptar los contenidos a las necesidades de la audiencia conlleva, entre otras cosas, a dar mayor cabida a 
contenidos, estrategias y actividades vinculadas a la adquisición de vocabulario, aspecto fundamental dentro del proceso de aprendizaje del inglés.

Esas adecuaciones curriculares "consisten en una secuencia de acciones sobre un currículo diseñado para una determinada población que conducen a la modificación de alguno o todos sus elementos" [7, p. 24], por lo que el docente singulariza y contextualiza la propuesta pedagógica de acuerdo a las limitaciones para el aprendizaje y la participación que observa en sus estudiantes.

En otras palabras, aun cuando se trata de un término utilizado recurrentemente en el campo de la educación inclusiva, se debe tener en cuenta que un bajo nivel de vocabulario -para efectos de aprendizaje de una lenguadebe considerarse como una limitación o barrera.

En consecuencia, para dilucidar como se atiende a las premisas de esta relación entre adquisición de vocabulario y aprendizaje del inglés el desarrollo de la presente investigación se enfocó en la manera como los docentes de inglés de octavo año de instituciones educativas oficiales de Guayaquil incorporan a sus planificaciones curriculares contenidos, estrategias y actividades hacia la ampliación del vocabulario.

\section{B. Justificación}

De acuerdo a [8] el diseño curricular es fundamental debido a que sirven como guía a los docentes de inglés en las instituciones educativas fiscales del país, pero para ello es indispensable incorporar los elementos necesarios para que los estudiantes puedan adquirir las destrezas que les faciliten dominar esta lengua extranjera.

En este particular, el presente trabajo está orientado a entrever como los contenidos dirigidos al aumento del vocabulario influyen en aprendizaje del idioma inglés en estudiantes de octavo año EGB se justifica desde diversas perspectivas. En primer lugar, en cierta forma, permitiría detectar las trabas y limitaciones que existen para el aprendizaje del inglés por parte de los estudiantes, lo que a la larga ayudaría a plantear soluciones al respecto.

En segundo lugar, es necesario ampliar la base teórica desde la cual se relaciona la ampliación del léxico con el nivel de aprendizaje alcanzado de una segunda lengua, comprendiendo que hasta ahora son mayores los enfoques teóricos que vinculan la adquisición de una lengua extrajera con el aspecto gramatical.

Asimismo, esta investigación se enmarca en el Plan Nacional de Desarrollo 2017 - 2021 Toda una Vida en su "Eje 1: Derechos para todos durante toda la vida" el cual contiene el "Objetivo 1: Garantizar una vida digna con iguales oportunidades para todas las personas" con su respectiva "Política 1.6: Garantizar el derecho a la salud, la educación y al cuidado integral durante el ciclo de vida, bajo criterios de accesibilidad, calidad y pertinencia territorial y cultural" [9].

Por otro lado, de acuerdo con [10], los profesores de instituciones educativas fiscales deben poner atención a los contenidos académicos de acuerdo con los conocimientos de los estudiantes y su entorno, para generar efectos positivos en el aprendizaje de este idioma; de allí que con la actual investigación se estaría buscando comprobar en qué medida los profesores de inglés de segundo año de las instituciones de Guayaquil atienden a esta premisa.
En definitiva, el presente trabajo de investigación busca contribuir en el proceso de enseñanza-aprendizaje del idioma inglés, identificando los factores adversos que perjudican o entorpecen este proceso; hallazgos a los que se busca llegar partiendo de la información recabada a través de la encuesta aplicada a un grupo de profesores, así como de la revisión bibliográfica.

\section{Objetivo general}

- Determinar la relación entre el nivel de inclusión en el diseño curricular de contenidos, estrategias y actividades dirigidas a la ampliación del vocabulario y el aprendizaje del idioma inglés de los estudiantes de octavo año EGB de colegios fiscales de Guayaquil.

\section{Objetivos específicos}

- Analizar la influencia de la ampliación de vocabulario en el aprendizaje del inglés.

- Medir la incorporación de contenidos, estrategias y actividades dirigidas a la ampliación del vocabulario de inglés en el octavo año EGB de colegios fiscales.

- Determinar el nivel de aprendizaje del inglés por parte de estudiantes de octavo año EGB de colegios fiscales.

- Describir la relación entre la incorporación de contenidos, estrategias y actividades que amplían el vocabulario de un idioma y el nivel de aprendizaje alcanzado.

\section{E. Hipótesis}

El aprendizaje del inglés de los estudiantes de octavo año EGB de colegios fiscales está relacionado con el nivel de inclusión en la planificación curricular de actividades vinculadas a la ampliación del vocabulario de dicho idioma.

\section{DESARROLLO}

\section{F. Currículum educativo}

A base de los conceptos propuesto por [4] se puede determinar que el currículum educativo es la forma práctica de implementar una teoría didáctica, a la enseñanza, y es considerado como un plan de acción que permite al docente desarrollarlo en el aula de clases con los estudiantes; además de ser una metodología de organización de las actividades educativas de acuerdo con los contenidos, técnicas y herramientas didácticas a utilizar.

La autora agrega que el objetivo de la educación es formar al ciudadano y forjar una sociedad a través de un currículum que procede como un proceso activo donde interviene un conjunto de elementos tales como factores sociales, objetivos, recursos, entre otros, los cuales interactúan para lograr ese objetivo educativo planteado. Esto involucra al currículum como un medio para esculpir un determinado prospecto educativo, que responda a las necesidades individuales, sociales y culturales. De esta manera, a través de la planificación y aplicación del currículum se respalda al sistema educativo para alcanzar el tipo de persona y de sociedad que la comunidad requiere de dicha institución.

Por último, la autora citada concluye en que es importante afrontar los problemas del currículum educativo comprendiendo su concepción y lo que este transmite. Lo esencial es que exista concordancia entre el 
proyecto educativo y la gestión del currículum. Para ello, se requiere que la educación plantee tres procesos indispensables: el desarrollo del individuo, la interrelación con la comunidad y la anexión de la cultura. El docente debe apuntar su accionar diario en la aplicación del currículum durante las horas de clase para dirigir los procesos de enseñanza-aprendizaje en los estudiantes. Asimismo, debe ajustarse a las directrices generales de la política educativa que normalizan la educación nacional.

Por otro lado, [11] comenta que la asignatura de inglés se debe de respaldar en un plan de estudios que les permita a los estudiantes alcanzar y practicar las habilidades de comunicación requeridas en el aprendizaje de este idioma. El plan de estudios puede contener actividades de escucha, lectura e inglés hablado, también actividades de vocabulario y gramática. Además, se requiere introducir lecciones sobre la pronunciación correcta. La práctica para tomar exámenes de inglés como el TOEFL, debe ser considerada en el plan de estudios, para preparar a los estudiantes a someterse a este tipo de certificaciones del idioma inglés.

\section{G. Adquisición de un segundo idioma}

De acuerdo con [4], se conoce como adquisición de una segunda lengua, al proceso por el cual la persona aprende e internaliza un idioma distinto a su lengua materna o nativa predominante. La adquisición de un segundo idioma en un proceso natural en infantes bilingües cuyos padres o familia se comunican a través de dos o más lenguas. En cambio, si este proceso se lleva a cabo en edades más avanzadas por parte del infante o adulto; está comprobado en la lingüística aplicada que este proceso de aprendizaje de una segunda lengua, contrasta sus fases del proceso de adquisición de la primera, debido a que se necesitan otro tipo de habilidades cognitivas.

Por lo tanto, el aprendizaje de un segundo idioma es un proceso más riguroso donde se necesita de mayor tiempo que la adquisición de la lengua nativa, en especial cuando estructuralmente el idioma propio y el segundo son muy distintos.

La investigación de [4] determinó que un niño comienza a pronunciar las primeras palabras de su lengua nativa en un proceso que conlleva una importante labor cognitiva de tipo fonológica; esto ayuda a que el niño asimile los sonidos de diversas pronunciaciones como actores del mismo tipo de unidad segmental o fonema. Por tal razón, la adquisición de un segundo idioma en muchos de los hablantes generalmente guarda un "acento" particular ya que, en la primera lengua, como en otras, las características fonéticas distintivas y secundarias para agrupar los sonidos en fonemas pueden variar.

No obstante, algunos individuos logran una fluidez y comprensión del segundo idioma, completamente indistinguible de su lengua nativa; esto ocurre especialmente cuando ambos idiomas son similares. Por otro lado, los niños bilingües que aprenden dos lenguas al mismo tiempo normalmente llegan a dominar ambos idiomas, debido a que el proceso de aprendizaje que aplican es distinto al procedimiento de adquisición de una segunda lengua cuando se ha aprendido a hablar una primera. El problema de aprender una segunda lengua durante la adultez radica especialmente cuando este idioma corresponde a otra familia lingüística y su aprendizaje es forzado.

Como menciona [10], la adquisición de una segunda lengua es un proceso más ventajoso, significativo y preciso cuando es utilizado en una situación de comunicación real, motivo por el cual es responsabilidad de los docentes el enseñar los fundamentos que requiere el idioma desde los primeros años de la etapa escolar.

La regularidad de aprendizaje para el lenguaje social es diferente al del aprendizaje del lenguaje académico. En contextos adecuados, el estudiante promedio de una segunda lengua demora alrededor de dos años en obtener habilidades de comunicación interpersonal elementales. Estas habilidades ingresan en el lenguaje cotidiano de contexto integrado que sucede entre interlocutores. Sin embargo, se demora entre cinco y siete años, bajo circunstancias aptas, en desarrollar la competencia lingüística académica cognoscitiva o lenguaje académico de poco contexto, a un grado similar al que estudian en su idioma nativo.

Estas autoras concluyen que la mayoría de los estudiantes de inglés, que aprenden el idioma como segunda lengua tienen problemas en ciertos aspectos, pese a que, aparentemente, dominan el inglés básico tradicional para la comunicación cotidiana y mantienen inconvenientes con la destreza lingüística académica cognoscitiva.

\section{H. Adquisición de vocabulario}

Los investigadores han propuesto diferentes teorías que intentan trazar el mapa de procesos implicados en la adquisición de vocabulario. Dependiendo de la postura adoptada por el investigador, diferentes hipótesis y, en consecuencia, diferentes experimentos han podido mostrar cuando tiene lugar la adquisición y cuándo no el aprendizaje de una segunda lengua a través de la ampliación del vocabulario.

La hipótesis de entrada de Stephen Krashen ha sido influyente en una línea de investigación, estudiando la efectividad de aprender grandes cantidades de vocabulario a través de una avalancha de aportes con la lectura extensa como enfoque de estudio. El modelo cuestiona la necesidad de una entrada estructurada, así como la necesidad de que los alumnos produzcan vocabulario recién encontrado para adquirirlo.

La hipótesis de entrada se basa en la premisa que los alumnos suelen tener éxito al inferir el significado del contexto. Sin embargo, los críticos han sostenido a menudo que este no es necesariamente el caso, y que los estudiantes pueden incluso no notar la palabra desconocida o hacer un esfuerzo por aprender su significado [12]. No obstante, la información comprensible es una de las fuentes más importantes para conocer nuevos vocabularios y, aunque no está en su forma completa, se considera que la hipótesis de entrada tiene sustento [3].

En cambio, otras teorías han mantenido la necesidad de métodos en los que los alumnos tengan la oportunidad de transmitir significado [13], notar nuevas palabras y su morfología en la comunicación [14], [15], y practicarlos a través de la producción [16]. Por consiguiente, se impone la necesidad de un aprendizaje centrado en el alumno.

El cambio en el enfoque del proceso de aprendizaje de enfoques orientados al maestro a enfoques centrados en el 
alumno durante las últimas décadas del siglo XX estuvo acompañado por la aparición del concepto de "competencia comunicativa" [17], [18] caracterizado por [19] como "capacidad comunicativa del lenguaje", un concepto que resalta las teorías del enfoque comunicativo para la enseñanza de idiomas extranjeros. En gran parte como resultado de la influencia del enfoque comunicativo, el logro de la competencia comunicativa se ha convertido en el objetivo principal de la enseñanza aprendizaje de una lengua extranjera.

[20] señala que el vocabulario es una parte importante de la lectura. Sin suficiente vocabulario, los lectores pueden adivinar las palabras desconocidas, pero no pueden comprender completamente los textos de lectura, o simplemente entenderlos vagamente, y muchas veces de manera incorrecta. Además, la capacidad de comprensión de los alumnos depende en gran medida de su conocimiento sobre el vocabulario. Se asume que tanto el vocabulario como el acervo posean los alumnos mostrarán la confianza en el manejo del idioma.

Aunque para [21] el lenguaje es un proceso social y el lenguaje surge en la vida del individuo a través de un intercambio continuo de significado con personas significativas, mejorar el vocabulario es uno de los objetivos de las clases de inglés como lengua extranjera, ya que esta actividad ayuda gradualmente a los alumnos a lograr la motivación y la confianza en sí mismos

Adquirir un vocabulario más amplio y profundo es primordial en la búsqueda de convertirse en un aprendiz competente de idiomas extranjeros. Se puede argumentar que la falta de conocimiento de vocabulario a menudo tiene un impacto más severo en la claridad y fluidez del lenguaje hablado y escrito que el conocimiento gramatical insuficiente o una pronunciación deficiente [22].

Se han establecido varios umbrales para tratar de precisar cuántas palabras en realidad debería aprenderse. [3] sostiene que, para comprender una variedad de textos, los estudiantes necesitan un léxico de alrededor de 80009000 familias de palabras. Se cree que este número es suficiente para una tasa de comprensión del $98 \%$ de una novela o un periódico.

Respecto a la práctica del método tradicional centrado en el uso del diccionario existen al menos tres puntos débiles con respecto al vocabulario: 1) errores comunes causados por la mala comprensión del significado de palabras o frases; 2) elegir palabras o sinónimos incorrectos; y 3) poca variación en la selección de vocabulario para la comprensión, etc.

[23] afirma que el uso de un diccionario bilingüe es una de las razones para elegir sinónimos incorrectos, mientras que [24] encontraron que el stock limitado de vocabulario es un gran obstáculo para que los estudiantes aprendan un idioma extranjero. El teórico señala que el aprendizaje de vocabulario es muy importante tanto para el desarrollo de la escritura de una segunda lengua como para la lectura, y por ello los docentes de inglés como segunda lengua deben reconocer y fomentar el aprendizaje de vocabulario.

I. Tipo Aprendizaje incidental vs Aprendizaje intencional

El aprendizaje incidental de vocabulario se puede describir como palabras que se adquieren al participar en una actividad con otro propósito que no sea aprender vocabulario, como leer un libro, escuchar en una conversación con un hablante nativo o tratando de decidir junto con un compañero el orden de los muebles en una habitación.

Por otra parte, el aprendizaje intencional se define como una actividad en la que aprender vocabulario es el propósito o énfasis de una tarea. Esto se puede hacer a través de una instrucción centrada en la forma o mediante actividades para el alumno, como memorizar listas de palabras. Por lo tanto, tal dicotomía es algo problemático como señalan [25], quienes argumentan que es importante reconocer el vocabulario adquirido como un subproducto de una actividad puede ser tanto accidental como intencionada.

Los teóricos sostienen que la intención solo puede ser medida a nivel de tarea, en forma de objetivos de tarea. Sin embargo, la intención también se encuentra en el nivel de aprendiz subordinado, con respecto a las elecciones hechas por el individuo para comprender y aprender la palabra designada, incluso si participa en una actividad de aprendizaje incidental.

Por lo tanto, el término incidental solo debe usarse para explicar el diseño de tareas en lugar de la situación de aprendizaje, ya que las estrategias que implementan los alumnos no se pueden conocer de antemano y no cuantificable [25]. Independientemente de cómo ocurran, se argumenta que las actividades centradas en la forma son esenciales para el aprendizaje y adquisición del vocabulario [26].

Estas actividades suelen dividirse en dos grupos: 1) Las enfocadas en formularios que denotan actividades descontextualizadas y no comunicativas que enfatizan forma léxica; y 2) las centradas en la forma, abarcando actividades en las que la forma se percibe como un resultado de la necesidad de captar el significado de una palabra, la necesidad de utilizar el vocabulario comunicación, o mediante la retroalimentación negativa del interlocutor [27].

\section{J. Profesores en el aprendizaje del idioma inglés}

De acuerdo con [5] en el sistema educativo es fundamental poseer docentes de calidad para perfeccionar la destreza del inglés, debido a que estos son los responsables de aplicar diariamente las políticas y actividades dispuestas por las instituciones estatales. No obstante, la enseñanza de este idioma ha presentado debilidades vinculadas tanto con la destreza del inglés por parte de los docentes como con la calidad de su formación.

Los autores agregan que los docentes en Latinoamérica presentan un bajo grado de destreza de esta lengua, por otro lado, sus políticas adoptadas ordenan un dominio desde los niveles B2 a C2 de acuerdo con el Marco Común Europeo de Referencia (MCER), las evaluaciones y análisis de diagnóstico determinan que la mayoría de los profesores de inglés poseen un desempeño muy inferior a estos estándares. Altos porcentajes de docentes se desempeñan difícilmente en los niveles requeridos para sus estudiantes, e inclusive en grados inferiores.

Actualmente existe una alta demanda de docentes de inglés en Latinoamérica, asimismo, las oportunidades de formación para los profesores son elevadas en la región, la calidad de esta formación es diversa, aunque deficiente. Si bien es cierto, se encuentran en el mercado sistemas de acreditación para asegurar la calidad de la formación, 
también existen un sin número de cursos no acreditados que continúan inscribiendo estudiantes e impartiendo cátedra. Además, existe una amplia gama en el empleo de requerimientos de ingreso o egreso, y pocos países poseen un contexto estandarizado. A esto se le suma la situación actual de las alternativas de desarrollo profesional para los profesores de inglés los cuales padecen problemas de valoración y prolongación.

A lo largo de la región se pueden encontrar oportunidades de capacitarse tanto localmente como en el extranjero. Al igual que las oportunidades han ido aumentando, existe muy poca evidencia de las consecuencias y el costo de estos programas; la gran variedad de cursos nuevos en la región también da una insuficiente progresividad a programas previos.

Estas debilidades, como los mencionan los autores, están asociadas a una efímera aplicación de políticas vinculadas con la formación de los docentes, lo que ha generado una deficiente calidad de los maestros de inglés; los profesores presentan un inferior grado de destreza de esa lengua y frecuentemente no cuentan con certificación o adiestramiento. Los países de la región deben asumir políticas que ayuden a optimizar la formación de sus docentes de inglés si desean tener un efecto positivo en la trasmisión efectiva de este idioma.

\section{K. El rol del docente}

Como menciona [1] la adquisición de una lengua extranjera como el inglés necesita de profesores debidamente capacitados en relación con metodologías adecuadas para un óptimo proceso de enseñanzaaprendizaje de este idioma, además el maestro debe tener las suficientes habilidades para reconocer a los distintos tipos de alumnos, sus maneras de aprendizaje, sus dificultades de aprendizaje, entre otros. Los alumnos utilizarán la lengua inglesa dentro de las aulas si el docente implementa las siguientes condiciones que se desprenden de los postulados y recomendaciones del citado autor:

- La planificación de los procesos necesarios para el empleo del segundo idioma, según el grado de los estudiantes y considerando que, normalmente, comprenden más de lo que están dispuestos a producir.

- Expresarse de la manera más precisa y compresible posible. Sustentar visualmente los argumentos y los correctivos a través de mímicas, multimedia, objetos, el empleo de la pizarra, entre otros.

- Adiestrar a los estudiantes a que utilicen técnicas idiomáticas en inglés. La enseñanza y la motivación del empleo de términos genéricos que optimizan su habilidad de comunicación.

- Acostumbrar a los estudiantes con las expresiones más frecuentes que se emplean en clases y su uso habitual y con sentido. Promocionar la habilidad de los alumnos para deducir la definición por medios lógicos como el contexto y las relaciones entre conceptos y frases.

- Si se aplica cada uno de los factores detallados previamente, se conseguirá ofrecer los escenarios apropiados que ayuden al empleo frecuente y práctica habitual del inglés, asimismo, se alcanzará a incluir al alumno en un aprendizaje continuo y progresivo.

\section{Rol de estudiante}

En la misma línea de [1] se determina que el rol del alumno es una de las transcendentales modificaciones que se ha conseguido en la enseñanza del idioma. Estos roles juegan un papel más dinámico debido a que serán los responsables del grado de desarrollo de las destrezas del idioma y son ellos quienes deben adoptar una iniciativa más frecuente durante las clases.

Para los alumnos que adquieren el idioma inglés como lengua extranjera uno de los escenarios de aprendizaje más primordiales es el aula de clases, donde se desarrolla el proceso de enseñanza-aprendizaje, convirtiéndose en el principal espacio de prueba y práctica que les proporciona el perfeccionamiento adecuado para que pueda desenvolverse y comunicarse fluidamente en este idioma.

Luego de haber explicado el rol que desempeñan profesores y estudiantes dentro del proceso de enseñanzaaprendizaje del idioma inglés como lengua extranjera, [1] agrega que estos dos elementos deben de llevar la clase en completa armonía, trabajando bajo directrices que destaquen el respeto y un elevado nivel de responsabilidad, simplemente una adecuada mezcla de estos dos factores proporcionará logros relevantes en el desarrollo de la habilidad comunicativa en el idioma inglés.

\section{METODOLOGÍA \\ M. Tipo de investigación}

El presente trabajo corresponde a una investigación de campo, transversal, no experimental y descriptiva, con un enfoque cuantitativo y con un diseño de aplicación tipo encuesta. Se lo considera de campo porque se realizaron encuestas a docentes de la asignatura de inglés en instituciones educativas fiscales de la zona 8 dentro de la ciudad de Guayaquil. Descriptiva porque ayudará a mejorar el proceso de enseñanza-aprendizaje del idioma inglés en instituciones educativas fiscales, ya que el problema se lo va a detallar tal y como se muestra en la realidad. No experimental, debido a que se realizó el trabajo sin manipular intencionadamente las variables. La investigación posee un enfoque cuantitativo, agregando un componente cualitativo para facilitar el análisis de la información en algunas variables permitiendo interpretar los resultados desde un contexto más amplio.

\section{N. Población y muestra}

La población de estudio está determinada en 2,983 profesores de inglés de instituciones educativas fiscales del cantón Guayaquil. Utilizando la fórmula para poblaciones infinitas, se obtuvo como resultado una muestra de 340 profesores de inglés, sin embargo, al aplicar el instrumento, participaron 469 docentes, por lo que se consideraron a todos los individuos que participaron en la investigación de campo.

\section{O. Técnicas e instrumentos de recolección de información}

Para la recopilación de información se utilizó la técnica de la encuesta y como instrumento un cuestionario estructurado conformado por 24 preguntas, en su mayoría cerradas, dirigido a los profesores de inglés que actualmente enseñan en las instituciones educativas fiscales del cantón Guayaquil. En concordancia con los objetivos establecidos, esto permitió identificar el problema planteado. Se elaboraron los respectivos formatos de investigación para la muestra seleccionada, los 
mismos que fueron revisados y aprobados por el Ministerio de Educación.

\section{RESULTADOS}

Se determinó que el principal problema que influye en el proceso de enseñanza-aprendizaje del inglés en estudiantes de octavo año de Educación General Básica en colegios fiscales de la ciudad de Guayaquil, es la disparidad en los contenidos académicos para los distintos niveles en los cuales se imparte el idioma inglés, especialmente por un enfoque excesivo en el aspecto gramatical, dejando de lado la adquisición y desarrollo de vocabulario.

En la mayoría de los casos los estudiantes ni siquiera recibieron formación previa para esta asignatura en sus currículos académicos durante sus primeros años de primaria, debido a que el programa de inglés está vigente a partir del periodo 2017 en el régimen costa; lo que supone un fuerte impacto y genera poca asimilación de este idioma en dichos estudiantes; los datos encontrados se muestran en las tablas descritas a continuación.

Como se puede observar en la Tabla I los profesores indican que los estudiantes de octavo año de colegios fiscales de Guayaquil poseen mayores dificultades en el aprendizaje del idioma inglés, debido a un contenido muy superior en comparación a los años anteriores, de donde provienen con una buena base, y se debe reforzar todo lo aprendido, no existiendo la debida adquisición de competencias para cada nivel.

TABLA I

PROBLEMAS DE CONTENIDO ACADÉMICO QUE ENFRENTAN LOS ESTUDIANTES POR AÑO DE EGB

\begin{tabular}{|c|c|c|c|c|c|c|c|c|}
\hline \multirow{2}{*}{\multicolumn{3}{|c|}{\begin{tabular}{l}
\multicolumn{1}{c}{ C) 7.1. En su } \\
experiencia como \\
docente ¿en qué año \\
de educación general \\
básica cree usted que \\
los estudiantes \\
encuentran mayor \\
dificultad para el \\
aprendizaje del \\
idioma inglés? Señale \\
solo uno \\
\end{tabular}}} & \multicolumn{5}{|c|}{$\begin{array}{l}\text { C) 8.2. Escasa adecuación } \\
\text { curricular a entorno de aprendizaje }\end{array}$} & \multirow[b]{2}{*}{ Total } \\
\hline & & & $\begin{array}{l}\text { Muy } \\
\text { bajo }\end{array}$ & Bajo & Medio & Alto & $\begin{array}{l}\text { Muy } \\
\text { alto }\end{array}$ & \\
\hline \multirow{8}{*}{$\begin{array}{l}\text { C) } \\
\text { 7.1. }\end{array}$} & \multirow{2}{*}{ 8vo año } & No. & 18 & 21 & 61 & 101 & 88 & 289 \\
\hline & & $\%$ & $6,2 \%$ & $7,3 \%$ & $21,1 \%$ & $34,9 \%$ & $30,4 \%$ & $100,0 \%$ \\
\hline & \multirow{2}{*}{ 9no año } & No. & 1 & 5 & 4 & 4 & 8 & 22 \\
\hline & & $\%$ & $4,5 \%$ & $22,7 \%$ & $18,2 \%$ & $18,2 \%$ & $36,4 \%$ & $100,0 \%$ \\
\hline & \multirow{2}{*}{$\begin{array}{l}\text { 10mo } \\
\text { año }\end{array}$} & No. & 10 & 10 & 51 & 38 & 49 & 158 \\
\hline & & $\%$ & $6,3 \%$ & $6,3 \%$ & $32,3 \%$ & $24,1 \%$ & $31,0 \%$ & $100,0 \%$ \\
\hline & \multirow{2}{*}{ Total } & No. & 29 & 36 & 116 & 143 & 145 & 469 \\
\hline & & $\%$ & $6,2 \%$ & $7,7 \%$ & $24,7 \%$ & $30,5 \%$ & $30,9 \%$ & $100,0 \%$ \\
\hline
\end{tabular}

De acuerdo con la prueba Chi-cuadrado, el vínculo entre el curso con mayor dificultad de aprendizaje del idioma inglés y contenido académico superior en comparación a años anteriores es significante en un 0.022 , esto es menor al margen de error del $5 \%$ por lo que la relación es aceptada. Ver TABLA II
TABLA II

PRUEBAS DE CHI-CUADRADO EN LA CORRELACIÓN DIFICULTAD DE APRENDIZAJE Y CONTENIDO ACADÉMICO SUPERIOR

\begin{tabular}{|l|r|r|r|}
\hline \multicolumn{4}{|c|}{ Pruebas de chi-cuadrado } \\
\hline & \multicolumn{1}{|c|}{ Valor } & \multicolumn{1}{|c|}{ df } & \multicolumn{1}{c|}{$\begin{array}{c}\text { Significación } \\
\text { asintótica (bilateral) }\end{array}$} \\
\hline $\begin{array}{l}\text { Chi-cuadrado de } \\
\text { Pearson }\end{array}$ & $17,944^{\mathrm{a}}$ & 8 & 0,022 \\
\hline $\begin{array}{l}\text { Razón de } \\
\text { verosimilitud }\end{array}$ & 15,775 & 8 & 0,046 \\
\hline $\begin{array}{l}\text { Asociación lineal } \\
\text { por lineal }\end{array}$ & 0,675 & 1 & 0,411 \\
\hline N de casos válidos & 469 & & \\
\hline
\end{tabular}

a. 2 casillas $(13,3 \%)$ han esperado un recuento menor que 5. El recuento mínimo esperado es 1,36 .

Debido al alto contenido académico en comparación a los años anteriores, los profesores de inglés de octavo año de EGB de colegios fiscales de Guayaquil aplican un menor esfuerzo en la enseñanza de vocabulario tal y como se muestra en la TABLA III detallada a continuación:

TABLA III

CONTENIDO ACADÉMICO MUY SUPERIOR CON ESCASO ENFOQUE EN VOCABULARIO

\begin{tabular}{|c|c|c|c|c|c|c|c|c|}
\hline \multicolumn{3}{|c|}{ C) 4.3. Vocabulario } & \multicolumn{5}{|c|}{$\begin{array}{l}\text { C) 8.2. Escasa adecuación } \\
\text { curricular a entorno de } \\
\text { aprendizaje }\end{array}$} & \multirow[t]{2}{*}{ Total } \\
\hline & & & $\begin{array}{l}\text { Muy } \\
\text { bajo }\end{array}$ & Bajo & Medio & Alto & $\begin{array}{l}\text { Muy } \\
\text { alto }\end{array}$ & \\
\hline \multirow{10}{*}{ C) 4.3 . } & \multirow{2}{*}{ Raramente } & No. & 3 & 0 & 2 & 0 & 0 & 5 \\
\hline & & $\%$ & $60,0 \%$ & $0,0 \%$ & $40,0 \%$ & $0,0 \%$ & $0,0 \%$ & $100,0 \%$ \\
\hline & \multirow{2}{*}{$\begin{array}{l}\text { Algunas } \\
\text { veces }\end{array}$} & No. & 1 & 8 & 11 & 7 & 11 & 38 \\
\hline & & $\%$ & $2,6 \%$ & $21,1 \%$ & $28,9 \%$ & $18,4 \%$ & $28,9 \%$ & $100,0 \%$ \\
\hline & \multirow{2}{*}{$\begin{array}{l}\text { Muchas } \\
\text { veces }\end{array}$} & No. & 7 & 11 & 44 & 47 & 32 & 141 \\
\hline & & $\%$ & $5,0 \%$ & $7,8 \%$ & $31,2 \%$ & $33,3 \%$ & $22,7 \%$ & $100,0 \%$ \\
\hline & \multirow{2}{*}{ Siempre } & No. & 18 & 17 & 59 & 89 & 102 & 285 \\
\hline & & $\%$ & $6,3 \%$ & $6,0 \%$ & $20,7 \%$ & $31,2 \%$ & $35,8 \%$ & $100,0 \%$ \\
\hline & \multirow{2}{*}{ Total } & No. & 29 & 36 & 116 & 143 & 145 & 469 \\
\hline & & $\%$ & $6,2 \%$ & $7,7 \%$ & $24,7 \%$ & $30,5 \%$ & $30,9 \%$ & $100,0 \%$ \\
\hline
\end{tabular}

Como se puede observar en la TABLA III los profesores indican que los estudiantes de octavo año de colegios fiscales de Guayaquil poseen una exigencia superior en la materia en comparación a los años anteriores, llegando a ese nivel sin la preparación necesaria. Por otro lado, los docentes implementan en menor medida ejercicios de refuerzo en vocabulario, pese a que los estudiantes 
provienen de un ambiente donde el contacto con palabras inglesas es inusual.

De acuerdo con la prueba Chi-cuadrado, el vínculo entre la frecuencia de implementación del vocabulario y contenido académico superior en comparación a años anteriores es significante en un 0.000 , esto es menor al margen de error del $1 \%$ por lo que la relación es aceptada. Ver TABLA IV

TABLA IV

PRUEBAS DE CHI-CUADRADO EN LA CORRELACIÓN ESCASA FRECUENCIA DE IMPLEMENTACIÓN DEL VOCABULARIO Y CONTENIDO ACADÉMICO SUPERIOR

\begin{tabular}{|l|r|r|r|}
\hline \multicolumn{4}{|c|}{ Pruebas de chi-cuadrado } \\
\hline & \multicolumn{1}{|c|}{ Valor } & \multicolumn{1}{c|}{ df } & \multicolumn{1}{c|}{$\begin{array}{c}\text { Significación } \\
\text { asintótica (bilateral) }\end{array}$} \\
\hline $\begin{array}{l}\text { Chi-cuadrado de } \\
\text { Pearson }\end{array}$ & $50,508^{\mathrm{a}}$ & 12 & 0,000 \\
\hline $\begin{array}{l}\text { Razón de } \\
\text { verosimilitud }\end{array}$ & 36,585 & 12 & 0,000 \\
\hline $\begin{array}{l}\text { Asociación lineal } \\
\text { por lineal }\end{array}$ & 12,666 & 1 & 0,000 \\
\hline N de casos válidos & 469 & & \\
\hline
\end{tabular}

a. 7 casillas $(35,0 \%)$ han esperado un recuento menor que 5 . El recuento mínimo esperado es, 31 .

Por consiguiente, uno de los problemas que enfrentan los estudiantes de octavo año de colegios fiscales de Guayaquil es la falta de un currículo de inglés durante los primeros años de primaria (2do a 5to grado EGB) centrado en la expansión del vocabulario a fin de facilitar una base sólida de conocimientos del idioma. Con ello, se asume que los profesores implementan ejercicios de vocabulario con menor frecuencia en las clases de inglés, como se puede observar en la TABLA V detallada a continuación:

TABLA V

ADAPTACIÓN CURRICULAR DE INGLÉS DURANTE LOS PRIMEROS AÑOS DE PRIMARIA Y AMPLIACIÓN DE VOCABULARIO

\begin{tabular}{|c|c|c|c|c|c|c|c|c|}
\hline \multirow{2}{*}{\multicolumn{3}{|c|}{ C) 4.3. Vocabulario }} & \multicolumn{5}{|c|}{$\begin{array}{l}\text { C) 8.4. Falta de un currículo de inglés } \\
\text { durante los primeros años de primaria } \\
\text { (2do a 5to grado EGB) que expanda el } \\
\text { vocabulario y facilite una base sólida } \\
\text { de conocimientos del idioma }\end{array}$} & \multirow{3}{*}{$\begin{array}{r}\text { Total } \\
\\
5\end{array}$} \\
\hline & & & $\begin{array}{l}\text { Muy } \\
\text { bajo }\end{array}$ & Bajo & Medio & Alto & $\begin{array}{l}\text { Muy } \\
\text { alto }\end{array}$ & \\
\hline \multirow{10}{*}{$\begin{array}{l}\text { C) } \\
4.3\end{array}$} & \multirow{2}{*}{$\begin{array}{l}\text { Rara- } \\
\text { mente }\end{array}$} & No. & 3 & 0 & 2 & 0 & 0 & \\
\hline & & $\%$ & $60,0 \%$ & $0,0 \%$ & $40,0 \%$ & $0,0 \%$ & $0,0 \%$ & $100,0 \%$ \\
\hline & \multirow{2}{*}{$\begin{array}{l}\text { Algunas } \\
\text { veces }\end{array}$} & No. & 7 & 7 & 14 & 4 & 6 & 38 \\
\hline & & $\%$ & $18,4 \%$ & $18,4 \%$ & $36,8 \%$ & $10,5 \%$ & $15,8 \%$ & $100,0 \%$ \\
\hline & \multirow{2}{*}{$\begin{array}{l}\text { Muchas } \\
\text { veces }\end{array}$} & No. & 10 & 24 & 44 & 33 & 30 & 141 \\
\hline & & $\%$ & $7,1 \%$ & $17,0 \%$ & $31,2 \%$ & $23,4 \%$ & $21,3 \%$ & $100,0 \%$ \\
\hline & \multirow{2}{*}{ Siempre } & No. & 18 & 26 & 71 & 89 & 81 & 285 \\
\hline & & $\%$ & $6,3 \%$ & $9,1 \%$ & $24,9 \%$ & $31,2 \%$ & $28,4 \%$ & $100,0 \%$ \\
\hline & \multirow{2}{*}{ Total } & No. & 38 & 57 & 131 & 126 & 117 & 469 \\
\hline & & $\%$ & $8,1 \%$ & $12,2 \%$ & $27,9 \%$ & $26,9 \%$ & $24,9 \%$ & $100,0 \%$ \\
\hline
\end{tabular}

Como se puede observar en la TABLA V, la falta de un currículo de inglés durante los primeros años de primaria influye en un nivel alto con la implementación de buen uso y manejo de vocabulario se repite con una frecuencia constante y repercute en el resto de los niveles educativos durante la enseñanza de este idioma.

De acuerdo con la prueba Chi-cuadrado, el vínculo entre la escasa frecuencia de implementación del vocabulario y la adaptación curricular del inglés durante los primeros años de primaria es significante en un 0.000 , esto es menor al margen de error del $1 \%$ por lo que la relación es aceptada. Ver TABLA VI

TABLA VI

PRUEBAS DE CHI-CUADRADO EN LA CORRELACIÓN FRECUENCIA DE IMPLEMENTACIÓN DEL VOCABULARIO Y LA FALTA DE UN CURRÍCULO DE INGLÉS

\begin{tabular}{|l|r|r|r|}
\hline \multicolumn{4}{|c|}{ Pruebas de chi-cuadrado } \\
\hline & \multicolumn{1}{|c|}{ Valor } & \multicolumn{1}{c|}{ df } & \multicolumn{1}{c|}{$\begin{array}{l}\text { Significación } \\
\text { asintótica (bilateral) }\end{array}$} \\
\hline $\begin{array}{l}\text { Chi-cuadrado de } \\
\text { Pearson }\end{array}$ & $44,898^{\mathrm{a}}$ & 12 & 0,000 \\
\hline $\begin{array}{l}\text { Razón de } \\
\text { verosimilitud }\end{array}$ & 37,924 & 12 & 0,000 \\
\hline $\begin{array}{l}\text { Asociación lineal } \\
\text { por lineal }\end{array}$ & 25,206 & 1 & 0,000 \\
\hline N de casos válidos & 469 & & \\
\hline
\end{tabular}

a. 7 casillas $(35,0 \%)$ han esperado un recuento menor que 5 . El recuento mínimo esperado es, 41 .

Contrariamente, el currículo de inglés durante los primeros años de primaria tiene una alta incidencia en la implementación de ejercicios de gramática durante las clases, como se puede observar en la TABLA VII detallada a continuación:

TABLA VII

CURRÍCULO DE INGLÉS DURANTE LOS PRIMEROS AÑOS DE PRIMARIA Y LA GRAMÁTICA

\begin{tabular}{|c|c|c|c|c|c|c|c|c|}
\hline \multicolumn{3}{|c|}{$\begin{array}{l}\text { C) } 4.4 . \\
\text { Gramática }\end{array}$} & \multicolumn{5}{|c|}{$\begin{array}{l}\text { C) 8.4. Falta de un currículo de } \\
\text { inglés durante los primeros años de } \\
\text { primaria (2do a } 5 \text { to grado EGB) que } \\
\text { expanda el vocabulario y facilite una } \\
\text { base sólida de conocimientos del } \\
\text { idioma }\end{array}$} & \multirow{3}{*}{$\begin{array}{r}\text { Total } \\
\\
12\end{array}$} \\
\hline & & & $\begin{array}{l}\text { Muy } \\
\text { bajo }\end{array}$ & Bajo & Medio & Alto & $\begin{array}{l}\text { Muy } \\
\text { alto }\end{array}$ & \\
\hline \multirow{10}{*}{$\begin{array}{l}\text { C) } \\
4.4 .\end{array}$} & \multirow{2}{*}{$\begin{array}{l}\text { Rara- } \\
\text { mente }\end{array}$} & \begin{tabular}{|l|} 
No. \\
\end{tabular} & 4 & 2 & 3 & 2 & 1 & \\
\hline & & $\%$ & $33,3 \%$ & $16,7 \%$ & $25,0 \%$ & $16,7 \%$ & $8,3 \%$ & $100,0 \%$ \\
\hline & \multirow{2}{*}{$\begin{array}{l}\text { Algunas } \\
\text { veces }\end{array}$} & No. & 6 & 11 & 27 & 21 & 5 & 70 \\
\hline & & $\%$ & $8,6 \%$ & $15,7 \%$ & $38,6 \%$ & $30,0 \%$ & $7,1 \%$ & $100,0 \%$ \\
\hline & \multirow{2}{*}{$\begin{array}{l}\text { Muchas } \\
\text { veces }\end{array}$} & No. & 13 & 24 & 50 & 47 & 40 & 174 \\
\hline & & $\%$ & $7,5 \%$ & $13,8 \%$ & $28,7 \%$ & $27,0 \%$ & $23,0 \%$ & $100,0 \%$ \\
\hline & \multirow{2}{*}{ Siempre } & No. & 15 & 20 & 51 & 56 & 71 & 213 \\
\hline & & $\%$ & $7,0 \%$ & $9,4 \%$ & $23,9 \%$ & $26,3 \%$ & $33,3 \%$ & $100,0 \%$ \\
\hline & \multirow{2}{*}{ Total } & No. & 38 & 57 & 131 & 126 & 117 & 469 \\
\hline & & $\%$ & $8,1 \%$ & $12,2 \%$ & $27,9 \%$ & $26,9 \%$ & $24,9 \%$ & $100,0 \%$ \\
\hline
\end{tabular}


Como se puede observar en la TABLA VII, es notable la presencia de un currículo de inglés durante los primeros años de primaria caracterizado por la implementación de ejercicios de gramática con una frecuencia reiterativa y constante durante la enseñanza de este idioma.

De acuerdo con la prueba Chi-cuadrado, el vínculo entre la frecuencia de implementación de la gramática y la falta de un currículo de inglés durante los primeros años de primaria es significante en un 0.001 , esto es menor al margen de error del $1 \%$ por lo que la relación es aceptada. Ver TABLA VIII

TABLA VIII

PRUEBAS DE CHI-CUADRADO EN LA CORRELACIÓN FRECUENCIA DE IMPLEMENTACIÓN DE LA GRAMÁTICA Y LA FALTA DE UN CURRÍCULO DE INGLÉS

\begin{tabular}{|l|r|r|r|}
\hline \multicolumn{4}{|c|}{ Pruebas de chi-cuadrado } \\
\hline & \multicolumn{1}{|c|}{ Valor } & \multicolumn{1}{|c|}{ df } & $\begin{array}{c}\text { Significación } \\
\text { asintótica (bilateral) }\end{array}$ \\
\hline $\begin{array}{l}\text { Chi-cuadrado de } \\
\text { Pearson }\end{array}$ & $33,853^{\mathrm{a}}$ & 12 & 0,001 \\
\hline $\begin{array}{l}\text { Razón de } \\
\text { verosimilitud }\end{array}$ & 32,959 & 12 & 0,001 \\
\hline $\begin{array}{l}\text { Asociación lineal } \\
\text { por lineal }\end{array}$ & 20,004 & 1 & 0,000 \\
\hline N de casos válidos & 469 & & \\
\hline $\begin{array}{l}\text { a. 5 casillas (25,0\%) han esperado un recuento menor que 5. El recuento } \\
\text { mínimo esperado es ,97. }\end{array}$
\end{tabular}

Como se ha evidenciado, entre las principales dificultades que poseen los estudiantes de octavo año EGB de colegios fiscales de Guayaquil en el proceso de enseñanza-aprendizaje en el idioma inglés; se encuentra principalmente la necesidad de la implementación de un currículo adecuado a su entorno socioeconómico, que abarque todos los niveles del sistema educativo desde la primaria, y extienda las competencias y contenidos acordes para cada nivel; ya que los resultados arrojados en el presente estudio indican que los docentes invierten más tiempo y esfuerzo a la enseñanza de la gramática, cubriendo el vocabulario a través del método tradicional (uso del diccionario), lo que revela la búsqueda de un aprendizaje predominantemente incidental.

\section{DISCUSIÓN}

Cabe destacar que la presente investigación como parte de la identificación de los problemas de enseñanza del idioma inglés se encontró una relación significativa entre la implementación de un currículo académico no adecuado a la realidad del estudiante; específicamente centrado en una enseñanza incidental del vocabulario.

La enseñanza del inglés en este nivel educativo se caracteriza por un alto contenido de exigencia en comparación a años anteriores, estas dificultades están estrechamente ligadas a la frecuencia de implementación y refuerzo por parte del docente de aspectos esencialmente gramaticales, mientras que las actividades orientadas a la adquisición y ampliación del vocabulario están delimitadas por el modelo tradicional (uso del diccionario).

Los resultados encontrados se vinculan con los de [8], quienes en una investigación realizada con niños de sexto grado que se inician en la enseñanza del idioma inglés se evidencia la presencia de dificultades principalmente en la comprensión de nuevas palabras, en este aspecto lo estudiantes tuvieron una calificación promedio inferior a 80, considerándose insatisfactorias. En tal sentido, se asume que los estudiantes provenientes de primaria tienen un vocabulario poco abundante y el mismo no es sujeto a métodos que procuren su ampliación,

En la misma línea de las destrezas del idioma inglés, [4] señala que son escasos los profesores que consiguen que sus alumnos efectúen conversaciones fluidas o que se expresen bien en esta lengua, por tal razón, destaca la importancia de ejecutar diversas actividades y estrategias durante las horas de clase para incrementar el vocabulario en este idioma; entre otras causas menciona la poca aplicación de ejercicios innovadores para hacer que los estudiantes adquieran y comprendan nuevos términos.

Asimismo, la autora también indica que no todos los alumnos desarrollan las micro habilidades que se necesitan para dominar la lengua inglesa. Como se puede observar, los resultados en esta investigación coinciden con lo expuesto al advertir la importancia de que se incorporen al currículo contenidos y actividades potenciadoras del desarrollo del vocabulario, trascendiendo de un aprendizaje incidental a uno intencional del vocabulario.

Por otro lado, [11] menciona que los problemas de aprendizaje del inglés en jóvenes estudiantes están ligados a la poca motivación, sustentándose esto en las deficiencias o insuficiencias en procesos básicos como la atención, la percepción que derivan en la escasa adquisición de vocabulario y con ello la presencia de obstáculos hacia la comprensión de la lectura y la transferencia de conocimientos e ideas.

En línea con tales argumentos de la autora citada, los resultados de la investigación actual indican que el problema del aprendizaje del inglés por parte de estudiantes de octavo año en los colegios fiscales seleccionados radica fundamentalmente en la falta de una adecuación curricular que tome en cuenta el contexto del estudiante, motivándolo a aprender el inglés para satisfacer sus necesidades.

Es fundamental pues que se revise, mejore y adapte la planificación curricular a las necesidades educativas de los estudiantes, especialmente en cuanto a vocabulario, lo que permitirá que estos se sientan motivados a aprender el idioma inglés.

En líneas generales, es necesario transcender en el empleo de un currículo centrado profusamente en actividades vinculadas al aprendizaje gramatical con apoyo del diccionario, y adaptarlo al entorno del estudiante incorporándole medios o recursos novedosos que despierten el interés del estudiante en adquirir $\mathrm{y}$ comprender los términos en inglés que actualmente se encuentran en sintonía con el avance tecnológico, principalmente las redes sociales e Internet.

\section{CONCLUSIONES}

Tras la realización de la presente investigación, se evidenció a través de la determinación de una relación causal que la principal problemática que enfrentan docentes y alumnos involucrados en la enseñanzaaprendizaje del idioma inglés, específicamente en el octavo año de instituciones educativas fiscales de Guayaquil, es la falta de un currículo adaptado a las necesidades del 
estudiante, así como equilibrado e innovador en cuanto a la incorporación de actividades propiciadoras de la ampliación del vocabulario.

Durante las últimas tres décadas, el aprendizaje y la enseñanza del vocabulario han atraído un interés significativo entre investigadores y docentes, ya que han llegado a reconocer la importancia y la posición fundamental que desempeña el conocimiento del vocabulario en el aprendizaje del inglés como lengua extranjera. Sin embargo, en el contexto de la enseñanza del inglés en Educación Secundaria en Ecuador las actividades dirigidas a ampliar el vocabulario se ignoran relativamente y no reciben la atención necesaria de los profesores y diseñadores de planes de estudios.

En dicho nivel educativo la mayoría de los docentes de inglés implementan el método tradicional de enseñanza de vocabulario como enumerar y definir nuevas palabras para luego pedir a los alumnos que memoricen los significados en inglés y español. En este entorno educativo centrado en el profesor, los estudiantes de inglés se convierten en receptores pasivos del lenguaje mucho más que en productores, y esto socava el desarrollo de su capacidad lingüística comunicativa.

Es un problema recurrente donde muchos estudiantes consideran que aprender vocabulario es un trabajo tedioso y culpan a su mala memoria porque no aprenden el inglés como segunda lengua, llegan a depender del aprendizaje incidental y encuentran el estudio individual aburrido e insuficiente. En este sentido, se ha comprobado que los estudiantes aprenden incluso eligiendo vocabulario de su propio entorno, lo que sucede desde ver su programa de dibujos animados favorito hasta la interacción dentro y fuera del aula de idiomas y, sobre todo, de las actividades del aula.

La enseñanza de vocabulario se convierte en una cuestión ineludible en el aprendizaje de un idioma, ya que la capacidad receptiva y comprensiva de los estudiantes a través de sonidos y símbolos depende en gran medida del volumen de vocabulario que poseen. Diversos estudios indican que, en general, los estudiantes no logran obtener una competencia satisfactoria en el uso del inglés y se ha detectado que su reserva de vocabulario es una de las razones subyacentes. Los resultados evidencian que el problema radica en la forma cómo los docentes planifican, presentan, practican y evalúan contenidos que involucran la enseñanza de esta micro habilidad.

Al mismo tiempo, la búsqueda de palabras en inglés / español en el diccionario, es la técnica dominante para adquirir nuevas palabras, y esta, según estudios previos, se ha percibido como frustrante tanto para los estudiantes como para los profesores, ya que la retención de vocabulario es baja en correspondencia con un igualmente bajo nivel de aprendizaje, lo que deja a los alumnos con dificultades con el inglés a lo largo de sus estudios.

\section{AGRADECIMIENTOS}

Este trabajo está financiado por Fondos Nacionales a través de FCT - Fundación para la Ciencia y la Tecnología dentro del ámbito del proyecto del CIEC (Centro de Investigación para Estudios Infantiles de la Universidad de Minho) bajo la referencia UID/CED/00317/2019.

\section{REFERENCIAS}

[1] M. Beltrán, El aprendizaje del idioma inglés como lengua extranjera. Boletín Virtual, 6(4), 91-98, 2017.

[2] C. Luna, Creación de un plan curricular para la asignatura de inglés, en el séptimo nivel de bachillerato del Colegio Francés de Quito, teniendo como base las artes. Quito: Universidad San Francisco de Quito - Colegio de Postgrados, 2011.

[3] P. Nation, The four strands. Innovation in Language Learning and Teaching, 1(1), 2-13, 2007. doi: 10.2167/illt039.0

[4] M. Arechúa, El diseño curricular formal de lengua extranjera y su incidencia en el proceso de enseñanza aprendizaje del idioma inglés a los estudiantes de octavo año de educación básica paralelo G del Colegio Fiscal El Empalme provincia del Guayas durante el periodo lectivo 2011-2012. Babahoyo: Universidad Técnica de Babahoyo - Facultad de Ciencias Jurídicas, Sociales y de la Educación, 2012.

[5] K. Cronquist, y A. Fiszbein, El aprendizaje del inglés en América Latina. El diálogo. Liderazgo para las Américas, 2017.

[6] J. Agudelo, J. Garay, y H. Morales, Propuesta para el mejoramiento del inglés en los estudiantes de $4 B$ del Colegio Antonio José de Sucre I.E.D, mediante la implementación de unidades didácticas multimediales. Bogotá: Universidad Libre Facultad Ciencias de la Educación - Departamento de Humanidades e Idiomas, 2009.

[7] Ministerio de Educación - Gobierno de la Ciudad de Buenos Aires, Buenos Aires, Argentina. Un currículum en común y diversificado Para todos los que enseñan y aprenden en la escuela, 1era ed. (2010). Accedido en: Octubre 20, 2020. [Online]. Recuperado de: https://www.buenosaires.gob.ar/areas/educacion/curricula/pdf/20 10 curriculum_encomun.pdf

[8] G. Torres, L. Vanega, y A. Britton, Análisis de los problemas que influyen en el aprendizaje del idioma inglés en los estudiantes de sexto grado en la provincia de Panamá. Revista de Iniciación Científica RIC, 4 (Especial), 119-121, 2018. doi:10.33412/revricv4.0.1833

[9] SENPLADES, Quito, Ecuador. Plan Nacional de Desarrollo 20172021 Toda una Vida, (2017). Recuperado de https://bit.ly/2Qf4YJC

[10] D. Barrezueta, y E. Merlo, Conocimientos heterogéneos del idioma inglés que presentan los estudiantes del sector rural y urbano al iniciar el octavo año de educación básica del Colegio Nacional Atahualpa. Ibarra: Universidad Técnica del Norte - Facultad de Educación, Ciencia y Tecnología, 2010.

[11] M. Sánchez, Las estrategias educativas y su incidencia en el proceso de enseñanza aprendizaje de la asignatura de inglés en los estudiantes del primer año del Colegio Fiscal Técnico "24 de Mayo" del cantón Quevedo, provincia de Los Ríos durante el periodo lectivo 2011 - 2012. Babahoyo: Universidad Técnica de Babahoyo - Facultad de Ciencias Jurídicas, Sociales y de la Educación, 2012.

[12] R. Oxford, y D. Crookall, Vocabulary learning: A critical analysis of techniques. TESL Canada Journal, 7(2), 9-30, 1990.

[13] P. Skehan, A framework for the implementation of task-based instruction. Applied Linguistics, 17(1), 38-62, 1996. doi: 10.1093/applin/17.1.38

[14] R. W. Schmidt, The role of consciousness in second language learning. Applied Linguistics, 11(2), 129-158, 1990.

[15] M. Long, The role of the linguistic environment in second language acquisition. In W. Ritchie \& T. Bhatia (eds). Handbook of second language acquisition. San Diego: Academic Press, pp. 413-468, 1996.

[16] M. Swain, y S. Lapkin, Problems in output and the cognitive processes they generate: A step towards second language learning. Applied Linguistics, 16(3), 371-391, 1995. doi:10.1093/applin/16.3.371

[17] D. Hymes, Two types of linguistic relativity. In Bright W (Ed), Sociolinguistics. 114-158. The Hague, The Netherlands: Mouton, 1966. Available: https://www.worldcat.org/title/sociolinguisticsproceedings-of-the-uclc-sociolinguistics-conference1964/oclc/2164408

[18] M. Canale, y M. Swain, Theoretical basis of communicative approaches to second language teaching and testing. Applied Linguisitcs. 1980,1(1):1-47.

[19] L. F. Bachman, Fundamental considerations in language testing. Oxford university press, 1990. 
[20] L. Q. Dong, "Beyond Reading: Vocabulary expansion and language use consolidation," presented at the 5th National VTTN ELT Conference, 2009. Retrieved from www.britishcouncil.org/lam_quang_dong.doc

[21] E. W. Stevik, Memory, meaning and method. Massachusetts: Newsbury House Publishers, 1976.

[22] T. Hedge, Teaching and learning in the language classroom. Oxford: Oxford University Press, 2000.

[23] M. H. Fallahzadeh, A Survey of the students and interns' EFL writing problems in Shiraz University of Medical Science. Asian EFL Journal: English Language Teaching and Research Articles, 9(3), 2007. Retrieved from Asian EFL Journal database

[24] J. M. Harmon, y K. D. Wood, Vocabulary teaching learning across disciplines. Research Summary, 2008. Retrieved from http://www.nmsa.org/Research/ResearchSummaries/VocabularyT eaching/tabid/1728/Default.aspx.

[25] A. Bruton, M. G. López, y R. E. Mesa, Incidental L2 vocabulary learning: An impracticable term? TESOL Quarterly, 45(4), 759768, 2011. doi: 10.5054/tq.2011.268061

[26] B. Laufer, y B. Rozovski-Roitblat, Incidental vocabulary acquisition: The effects of task type, word occurrence and their combination. Language Teaching Research, 15(4), 391-411, 2011. doi: $10.1177 / 1362168811412019$

[27] N. Shintani, The effect of focus on form and focus on forms Instruction on the acquisition of productive knowledge of L2 vocabulary by young beginning-level learners. TESOL Quarterly, 47(1), 36-62, 2013. doi: 10.1002/tesq.54

[28] L. Arévalo, J. Del Valle, C. Ñancucheo, R. Ortiz, y D. Quidel, La enseñanza del idioma inglés a temprana edad: Su impacto en el aprendizaje de los estudiantes de escuelas públicas. Revista De Comunicación Vivat Academia, XVII (129), 34-56, 2014.

[29] P. Tejeda, y A. Niebles, Análisis de algunos factores socioculturales en la enseñanza de un idioma extranjero. Estudios Pedagógicos, XLII (Especial), 31-39, 2016.

[30] D. Díaz, Factores de dificultad para el aprendizaje del inglés como lengua extranjera en estudiantes con bajo rendimiento en inglés de la Universidad Icesi. Santiago de Cali: Universidad ICESI - Centro de recursos para el aprendizaje, 2014. 